\title{
II.1 \\ Gastrine et omeprazole
}

B. MONGES

Marseille

L'OMEPRAZOLE chez I'homme et chez le rat entraine une achlorhydrie et une hypergastrinémie. L'administration à long terme est accompagnée d'une hyperplasie des cellules argyrophiles fundiques et de l'apparition de tumeurs carcinoïdes fundiques chez le rat. Les auteurs (* A 76) montrent qu'une administration prolongée d'OMEPRAzOLE entraîne un doublement du nombre de cellules à gastrine et a un effet trophique sur l'ensemble de la muqueuse gastrique. Ce dernier effet pourrait contribuer à la cicatrisation des ulcères. Par ailleurs (* A 175), à l'arrêt du traitement, ces effets sont réversibles : l'hyperplasie des cellules argyrophiles régresse après un certain délai, ainsi que 1 'hypergastrinémie.

$\mathrm{Au}$ cours du symposium de recherche sur les facteurs peptidiques de croissance, STEPHEN J.BRAND (BOSTON) montre que la sécrétion de gastrine et l'expression du gène de la gastrine sont stimulées par le TGF alpha (Transforming Growth Factor). Au niveau gastrique, le TGF alpha est sécrété par les cellules pariétales qui produisent également l'acide chlorydrique. L'OMEPRAZOLE, qui blóque la sécrétion d'acide chlorydrique au niveau des cellules pariétales, n'agit pas sur la sécrétion du TGF alpha qui continue à stimuler la production de gastrine. Ainsi commencent à se préciser les mécanismes complexes d'action de 1 'OMEPRAZOLE.

Par ailleurs, au cours du même symposium, ROBERT J. COFFEY (NASHVILLE), suggère que la maladie de MENETRIER pourrait être due à une dérégulation du TGF alpha. 